\title{
Sobre la poética y la crítica Literaria de José Gorostiza
}

\begin{abstract}
A L examinar la obra de José Gorostiza, lo primero que notamos es su escasa producción literaria, extendida a través de muchos años. ${ }^{1}$ En particular durante la juventud de Gorostiza, esto no puede ser debido a falta de devoción por la literatura, ya que ésta constituía entonces el objeto de su mayor interés. Por lo contrario, nos revela una actitud perfeccionista ante su obra. Desde el principio, nuestro autor selecciona y lima con cuidado lo que juzga digno de publicación. Jaime Torres Bodet, su compañero literario, lo nota varias veces: "Producía poco, no por esterilidad... sino por ansia de clásica perfección." "Se defendía de los sentidos limitándolos y procurando filtrarlos conscientemente." $\mathrm{Y}$ Xavier Villaurrutia, otro amigo del grupo "Contemporáneos", nota que Gorostiza excluye de su primer libro todos los poemas que considera inferiores; el libro constituye, en palabras de Villaurrutia, "una antología de cuanto
\end{abstract}

1 Gorostiza nace en Villahermosa, Tabasco, el 10 de noviembre de 1901; de joven se traslada a la ciudad de México. En 1920 uno de sus poemas aparece en una antología; en 1925, Gorostiza publica su primer libro de possía, Canciones para cantar en las barcas. Ya está enseñando literatura en la Universidad Nacional de México en 1929, e historia en la Escuela Nacional de Maestros en 1932. Mientras tanto, se une al grupo que publica la revista Contemporáneos (de donde viene el nombre con el cual llega a conocerse este grupo); entre 1928 y 1930 , nuestro autor publica dos poemas y cuatro reseñas en esta revista, más dos artículos en otra parte. Luego consigue empleo en el gobierno, desempeñando varios cargos hasta llegar a su actual puesto de Subsecretario de Relaciones Exteriores. A intervalos, continúa publicando: un poema aparece en 1936, cuatro más en 1938-1939. Este último año marca también la aparición de su segundo libro, el poema Muevte sin fin. Desde entonces Gorostiza ha publicado un poema, una carta abierta de crítica literaria y un largo ensayo sobre su teoría poética (su discurso de recepción como Miembro Correspondiente de la Academia Mexicana de la Iengua en 1955). Véase Jaime Torres Bodet, Tiempo de arena (México, 19.55), pp. 80-81, 184-185; también Who's Who in Latin America, 3d ed. (Stanford, Calif., 1946), parte I, p. 53.

2 Torres Bodet, pp. 81, 185. 
mejor decidióse, hasta entonces, a escribir."' El joven Gorostiza ya se nos revela como artista consciente en busca de la perfección. 4

Nuestro escritor estudia con ahinco, deseando aumentar y profundizar sus conocimientos. El primer ensayo que publica revela su interés en la estética; en él Gorostiza compara la poesía y la pintura como medios de creación.5 Como confesará luego, examina muchas teorías poéticas, desde Platón a Valéry. ${ }^{6}$ Pero también lee poesía; muy consciente de la herencia poética española, se siente atraído por la poesía tradicional, por Lope de Vega y por Góngora (debemos recordar que la década de I920 marca el redescubrimiento de éste). Notamos ya el interés de Gorostiza en la forma cuando escribe: “Qué disciplina más severa y más dentro del gusto contemporáneo que la de Góngora? ¿Qué mejor espejo de poesía?"' Pero a pesar de todas sus lecturas, el escritor pone cuidado en no dejarse llevar por la fácil imitación; elimina cuidadosamente de su primer libro todos los poemas que revelan influencias claras. ${ }^{8}$ Rechazando originalidad superficial y fácil imitación, Gorostiza se impregna en sus tradiciones y luego se esfuerza en producir una obra a la vez nítida y personal.

Esta combinación de estudio y disciplina es una de las cualidades más notables de todos los miembros del grupo "Contemporáneos", con el cual Gorostiza se identifica. El estudio de autores hispánicos y extranjeros es un recurso empleado por estos escritores para lograr un carácter menos provinciano y más profundo en sus obras. Acentúan valores estéticos más que sociales, y tienden a esquivar temas locales, lo que les ha valido mucha crítica enemiga:9 Sin embargo, tal vez lo que más les distingue es que ponen el énfasis en los fines attísticos y universales de la poesia, y escri-

3 Xavier Villaurrutia, "Un poeta" [fechado 1926], Textos y pretextos; literatura, drama, pintura (México, 1940), p. 79.

4 Más tarde, los cargos diplomáticos de Gorostiza le han impedido dedicar tiempo a la literatura, según comunicó al autor en una entrevista en México el 22 de junio de 1959. Indicó que llegó una época en que tuvo que escoger entre dos vocaciones, y no pudo rechazar la oportunidad de servir a su patria. $Y$ subrayó el carácter diferente de sus intereses sociales y literarios, rechazando la literatura social como una posible combinación de ambos. Véase también José Gorostiza, "Misión de la Academia", inédito discurso de recepción como Miembro Correspondiente de la Academia Mexicana de la Lengua, 1955, p. vi.

5 Véase José Gorostiza, "De la pintura nueva", Universidad, V (febrero 1928), 32-35.

16 Véase José Gorostiza, "Notas sobre poesía", Estaciones, III (primavera 1.958), 3.

"7' José Gorostiza, "Escalera", Contemporáneos, IV (julio 1929), 343.

8 Villaurrutia obscrva: "[En Canciones] las influencias ineludibles que ayudaron a conformar su espíritu apenas se muestran". Villaurrutia, "Un poeta". p. 79 .

- Véase Raúl Leiva, Imagen de la poesia mexicana contemporánea (México 1959), p. 77; Andrés Henestrosa, "Veinticinco años de poesía mexicana", Letras de México, III (15 de abril de 1942), 5-6; y Elías Nandino, "Panorana actual de la poesía mexicana", Universidad de México, V (junio 1951), 4-6. 
ben obras que tienen significado más allá de un solo lugar y de una sola época. Al mismo tiempo, su erudición y búsqueda de la universalidad protege a estos autores de la poesía irracional y sensacionalista de moda entonces. El alto fin que la poesía tiene para ellos les lleva a poner mucha atención en la construcción del poema y en los medios técnicos empleados para alcanzar el deseado significado exacto. ${ }^{10}$

Aparte de estas características, cada autor del grupo desarrolla sus propios temas y estilo; la obra creadora de cada uno difiere tanto en esencia que no podemos considerar su producción como una colección homogénea. Lo que les une no es un estilo o una forma, sino más bien un ideal de lo que la poesía debe tratar de lograr; en palabras de Villaurrutia, tienen "un concepto claro del arte como algo sustantivo y trascendente."11 La obra crítica de Gorostiza al examinar esta función trascendente de la poesía, aclara para nosotros la posición de este grupo y lo que ha logrado realizar. ${ }^{12}$

Después de delinear brevemente la actitud general de Gorostiza ante su obra y el carácter de "Contemporáneos", podemos ahora pasar a examinar con más detalle las ideas críticas y la poética de nuestro autor.

Al estudiar la obra crítica de Gorostiza, se puede notar una señalada estabilidad; sus ideas centrales permanecen casi igualcs entre I928 y I960. Y cualquier cambio menor que se observa resulta ser el desarrollo de ideas sostenidas previamente, o una ligera modificación de énfasis o enfoque.

Gorostiza considera el arte - y la poesía en particular-como poseedor de un tipo muy especial de significado. En 1932 lo caracteriza asi: "El arte es inconfundiblemente él mismo, y no deja de serlo para subordinarse a la filosofía, a la ciencia, o a los intereses económicos o políticos de una comunidad." 13 y continúa: "El arte no puede tener otro fin que él mismo. La teoría del arte por el arte es filosóficamente correcta. Pero si cumple su fin --esto es, si se cumple él mismo, si existe, si es en

10 Véase Frank Dauster, Breve bistoria de la poesia mexicana (México, 1956), pp. 149-150; del mismo crítico, "Notas sobre Muerte sin fin" (Revistat Iberoamericana, vol. XXV, No. 50, julio-diciembre, 1960, pp. 273-288); José Luis Martinez, Literatura mexicana siglo XX (1910-1949) (México, 1950), I, pp. 29-33; y Antonio Castro Leal, La poesía mexicana moderna [discurso ante la Academia Mexicana de la Lengua] (México, 1953), p. 30. p. 17.

11 Xavier Villaurrutia, La poesía de los jóvenes de México (México, 1924),

jis La revista del grupo, Contemporáneos (junio, 1928-diciembre 1931), es el vehículo por medio del cual cada uno concretiza su impulso creador ante el fondo de comunes ideales poéticos.

13 José Gorostiza, "El teatro de orientación", leído en la inauguración de la temporada teatral, el 28 de junio de 1932; Examen. No. 2 (septiembre, 1932), 21. 
verdad el arte- propagará fatalmente los ideales humanos de una época."14 Nuestro autor nunca se aparta de esta visión del arte como un modo independiente de conocimiento; al contrario, ésta llega a ser la base de su teoría poética. En todos sus escritos críticos, lo vemos esforzándose por subrayar y definir más la originalidad del arte y de la poesía, y de impedir que sean confundidos con otras disciplinas. ${ }^{\text {15 }}$ Gorostiza sin embargo no llega al extremo de divorciar la poesía de las preocupaciones humanas. Para él la poesía es justamente un modo único de penetrar y representar lo humano, y no un escape de ello. Cuando logra su mejor éxito, le da al hombre un conocimiento de los grandes problemas con los cuales éste tiene que enfrentarse. Dice nuestro crítico: "La poesía, para mí, es una investigación de ciertas esencias - el amor, la vida, la muerte, Dios-que se produce en un esfuerzo por quebrantar el lenguaje de tal manera que, haciéndolo más transparente, se pueda ver a través de él dentro de esas esencias." 16

La singularidad de la poesía depende del hecho de que usa el lenguaje de un modo creador. En contraste con el filósofo, con quien comparte la búsqueda de significados esenciales, el poeta no usa las palabras para analizar ideas lógicamente. Antes bien lleva las palabras a significados más profundos: "La poesía, al penetrar en la palabra, la descompone, la abre como un capullo a los matices de la significación."17 El poeta hace que las palabras comuniquen matices y emociones intangibles, $y$ al lograrlo da a su obra profundidad y originalidad. Y su esfuerzo debe de estar constante y conscientemente dirigido a obtener aún más significado encontrando la palabra y la frase exacta. ${ }^{18}$

De estas ideas proviene la visión que Gorostiza tiene de la belleza que debe lograr la poesía. No es de ningún modo una copia de lo bello en la naturaleza, sino más bien algo original que nace de la lucha del poeta con las palabras. Si usa éstas con éxito, el artista ha creado una nueva hermosura y ha enriquecido al mundo con ella: "La belleza manifestada por la poesía no la toma ésta del mundo exterior, no es la belleza natural de la nube o de la flor, sino la belleza artificial, poética, que la poesía presta transitoriamente, para sus propios fines, a la rosa y a la nube."19

Con su modo de ver la poesía como singular y trascendente, Gorostiza desconfía de la poesía social. Aunque los temas sociales pueden lle-

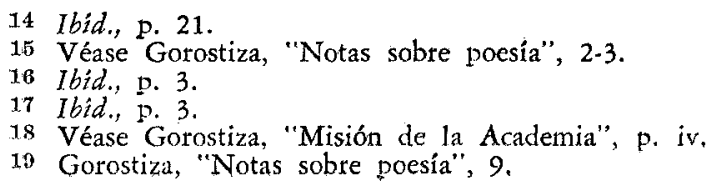


var a la poesía si van dotados de universalidad, los escritos sociales no tendrán valor poético si el tema político resulta más importante que la belleza creada por la obra. Y aquí, paradójicamente, Gorostiza acusa a los defensores de la poesía social de algo de que ellos lo habían acusado a él: falta de preocupación por los valores humanos. ${ }^{20}$ Si sus obras subrayan temas locales más que cualquier otra cosa, están ignorando el objetivo poético de lograr un conocimiento de problemas más universales.24

Pero Gorostiza también contradice a aquellos defensores de un arte puro que sostienen, como Valéry, que el poeta debe de reducir su poema a sólo esencias de puro arte. Nuestro escritor está de acuerdo con Valéry en la necesidad de buscar el lenguaje exacto para la expresión poética. Pero se niega a ver el trabajo del poeta como trabajo purgativo, dirigido a eliminar de la obra elementos no poéticos. Los hombres no pueden separarse de sus circunstancias, y las necesitan como método de comunicación; si la poesía eliminase todo lo que no fuese esencia, muy poco quedaría. $Y$ el arte "si se expresa deja de ser puro y si no se expresa deja de ser arte." "2i2 La función del poeta consiste en usar experiencias humanas como material para la creación de una obra universal, y no perderse en la caza de imposibles abstracciones. Aquí Gorostiza se sitúa cerca del Abate Brémond, quien sostuvo que el momento de poesía inefable ocurre, en medio de muchos otros elementos, en un buen poema - y no es el resultado de un proceso de purgación. ${ }^{23}$

Las ideas de Gorostiza sobre la poesía compleja y difícil están muy de acuerdo con su punto de vista general. Ya que el poema debe de llevar siempre hacia un significado profundo, el poeta tiene que librarse de confusión sin propósito, que simplemente impide que el lector vea el valor de la obra. Aunque la complejidad puede resultar valiosa si se usa para enriquecer el sentido del poema, las complicaciones supérfluas deben de evitarse."21 Aquí Gorostiza se opone a aquellos "estridentistas" y demás poetas de su generación que cultivan la extravagancia y la oscuridad solamente por crear efectos novedosos.

En su ensayo más reciente, Gorostiza introduce una nueva idea, que sin embargo no va en contra de sus principios críticos. Se queja de la falta

20 Véase nota 9.

121 Estas ideas fueron expresadas por Gorostiza en la entrevista concedida al autor el 22 de junio de 1959.

122. Gorostiza, "El teatro de orientación", 21.

23 Véase Alberto Monterde, La poesía pura en la lirica española (México; 1953), capítulos 1 y 2.

24 José Gorostiza, carta a Bernardo Ortiz de Montellano, publicada en el libro de éste. Una botella al mar (México, 1946), pp. 26-72. 
de poemas largos en la literatura contemporánea, e indica las limitaciones de una colección de poemas cortos: "Y en el libro podrá haber cierta uniformidad de emoción y de estilo. . .; pero el libro no mostrará, a su vez, la unidad de construcción que nos agrada encontrar en un libro. La suma de treinta momentos musicales no hará nunca el total de una sinfonía." "25 Mientras que un poema corto se limita por lo general a un solo tema o inspiración, en una obra más larga podemos encontrar una mayor cantidad de aspectos organizados en un esquema total de significado. Gorostiza, con su énfasis en la universalidad de la poesía, prefiere un poema de mayor alcance y aspiración, ya que tendrá mayor probabilidad de lograr una importante visión total, menos limitada a transitorios detalles particulares.

Nuestro escritor rechaza, finalmente, una poesía dirigida exclusivamente a las sensaciones. Ya que éstas son transitorias, no pueden formar la base de la poesía, que busca valores universales y se extiende más allá de un tiempo particular. ${ }^{26}$ En general, Gorostiza sistemáticamente rechaza cualquier interpretación del arte que lo subordinaria a otras disciplinas o lo privaría de una función cósmica. Su obra en prosa constituye un esfuerzo por afirmar y examinar la trascendencia de la creación literaria. El único cambio que notamos a través del tiempo es una creciente humildad: el crítico se muestra cada vez menos deseoso de imponer su opinión como norma absoluta. ${ }^{127}$ Gorostiza se da cuenta de que el poema es mortal y que puede perder su significado cuando cambios de época y estilo lo hacen inasequible al lector; sin embargo, se siente cada vez más seguro de la importancia del trabajo del poeta, que trata de extender por algún tiempo el significado de su mundo: "Nadie sino el Ser Unico más allá de nosotros, a quien no conocemos, podría sostener en el aire, por pocos segundos, el perfume de una violeta. El poeta puede - a semejanza suya- sostener por un instante mínimo el milagro de la poesía. Entre todos los hombres, él es uno de los pocos elegidos a quien se puede llamar con justicia un hombre de Dios."'28 En último término el poeta, al descubrir un modo de expresar lo universal en lenguaje humano y hacerlo asequible al lector a través de tiempo y espacio, añade algo nuevo al mundo y paralela el trabajo del Supremo Creador.

Al tratar de valorar la obra crítica de Gorostiza, uno no puede dejat de notar su aspecto humano; en todos sus ensayos se puede percibir la lu-

25 Gorostiza, "Notas sobre poesía", 9.

126 Escribe el crítico: "Lástima que no se puede fundar un arte verdadero en una sensación". Gorostiza, carta a Ortiz de Montellano, Una botella al mar, pp. $27-28$.

127 Gorostiza, "Notas sobre poesia", 3-4.

28 Ibid., p. 11. 
cha de un hombre - un poeta-por aseverar, explicar y justificar la importancia que la poesía tiene para él, y que él siente que debería de tener para todo el mundo. Su crítica no consiste en una fría búsqueda de categorías y definiciones, sino más bien en el esfuerzo humilde pero tenaz del artista por ayudar a otros a ver la belleza de su arte. Como él mismo escribe: "Pero este hombre necesita, él sí, de la poesía; que sople sobre su vida y la embellezca; que la salve de los tremendos infortunios que la amenazan y la haga digna de ser llevada con orgullo sobre los hombros."

Su amor a la poesía lleva a Gorostiza a afirmar su independencia y trascendencia; esta afirmación forma, como hemos visto, la base de su crítica. Para nosotros constituye el aspecto más valioso de su obra en prosa; por medio de ella recuerda a poetas y lectores que deben mantener altos sus ideales, guardarse de ser desviados por experiencias personales insignificantes o por crítica mezquina, y valerse de la poesía en la búsqueda de significados más permanentes. $Y$ al explicar la belleza humanamente creada que nos presenta la poesía, Gorostiza desmiente el muy común rebajamiento de este arte al nivel de imitación fotográfica.

El interés en los valores profundos también le permite ver a nuestro crítico los puntos débiles de algunas teorias poéticas, y le suministra pruebas contra ellos. Tomando parte en la disputa entre poetas "puros" y "sociales", muestra eficazmente cómo cada grupo puede alejar a la poesía de la universalidad - éste poniendo el énfasis en valores transitorios, aquél reduciendo demasiado el alcance humano de la poesía. $\mathrm{Y}$ al negarse a aceptar novedades exageradas, Gorostiza percibe las limitaciones de muchos de sus compañeros poetas: extravagancias sin significado, halagos a las sensaciones, excesivas fragmentación de la obra. Su más amplia perspectiva le permite rehuir muchos problemas de nuestra época.

El punto débil de la obra crítica de nuestro escritor sería, acaso, que su idealismo lo lleva hacia una excesiva abstracción. Cuando habla, por ejemplo, de la "sustancia poética" que ve presente en las grandes obras de arte, se aparta del significado concreto de los poemas en favor de generalizaciones vagas. ${ }^{30} \mathrm{Y}$ aunque nos presenta una visión muy atractiva de la belleza

29 Ibid. p. 10

30 La poética de Gorostiza nos recuerda las ideas de Juan Ramón Jiménez, cuya obra nuestro escritor sin duda conocía. Ambos van llevados, por su devoción a la poesía, a afirmar las cualidades trascendentes de ésta, su función de creación más bien que de imitación, y la necesidad de buscar la perfección al escribir poemas. Ambos también tienden a la excesiva abstracción. Véase Mercedes Pesado, "Influencias de Juan Ramón Jiménez en el grupo de Contemporáneos", tesis magistral inédita para la Universidad Femenina, México, 1949, pp. 21-24. Este estudio indica algunos interesantes puntos de contacto entre Juan Ramón y los 
creada por la poesía, nunca trata en detalle de cómo ésta difiere de la belleza natural, ni de cómo el poeta puede lograr, por medios naturales, la creación de un nuevo tipo de intuición no basado en la naturaleza. En resumidas cuentas, la crítica de Gorostiza nunca llena el vacío entre la poesía como ideal de creación y la poesía como lucha del hombre con las palabras - problema que ha causado dificultades a muchos otros críticos. ${ }^{31}$ Sin embargo, y para hacerle justicia, hay que recordar que Gorostiza no está tratando de construir un sistema cerrado de crítica, sino más bien de comunicarnos la importancia de la poesía, lo cual hace clara y cumplidamente.

Grinnell College, Iowa

ANDREW P. DEBICKI

poetas mexicanos, aunque desafortunadamente contiene muchas generalizaciones discutibles y citas no asimiladas.

31 Sobre este asunto véase Amado Alonso, Materia y forma en poesía (Madrid, 1955), pp. 11-60; este crítico considera a la intuición poética como algo que emerge de la relación entre emociones personales y la realidad externa. Compárese con otros puntos de vista en Carlos Bousoño, Teoría de la expresión poética, 2 a. ed. (Madrid, 1956), pp. 20-61; y Pedro Salinas, Reality and the Poet in Spanish Poetry (Baltimore, 1940).

32 Véase Gorostiza, "Notas sobre poesía", 3-4. La apreciación de la crítica de Gorostiza nos ayuda indudablemente a comprender su poesía. Sabiendo que rechaza una poesía superficial, cuidaremos de buscar significados más profundos en los nítidos poemas de Canciones para cantar en las barcas. Y conociendo la aversión del poeta a complicaciones sin sentido, seguiremos estudiando el difícil poema Muerie sin fin, seguros de que la forma se usą con un propósițo. 